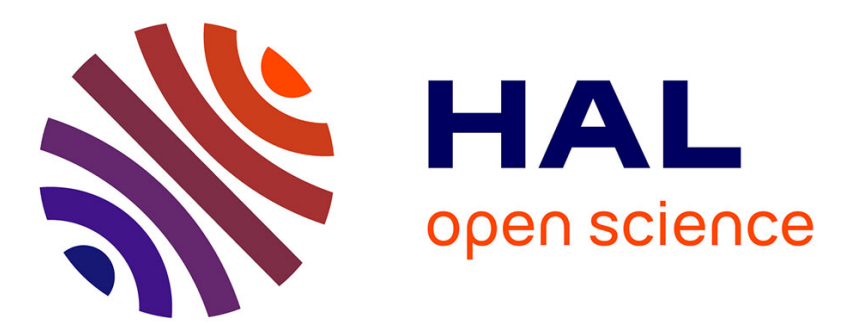

\title{
Nevanlinna counting function and Carleson function of analytic maps
}

Pascal Lefèvre, Daniel Li, Hervé Queffélec, Luis Rodriguez-Piazza

\section{To cite this version:}

Pascal Lefèvre, Daniel Li, Hervé Queffélec, Luis Rodriguez-Piazza. Nevanlinna counting function and Carleson function of analytic maps. 2009. hal-00375955v3

\section{HAL Id: hal-00375955 \\ https://hal.science/hal-00375955v3}

Preprint submitted on 17 Dec 2009

HAL is a multi-disciplinary open access archive for the deposit and dissemination of scientific research documents, whether they are published or not. The documents may come from teaching and research institutions in France or abroad, or from public or private research centers.
L'archive ouverte pluridisciplinaire HAL, est destinée au dépôt et à la diffusion de documents scientifiques de niveau recherche, publiés ou non, émanant des établissements d'enseignement et de recherche français ou étrangers, des laboratoires publics ou privés. 


\title{
Nevanlinna counting function and Carleson function of analytic maps
}

\author{
Pascal Lefèvre, Daniel Li, \\ Hervé Queffélec, Luis Rodríguez-Piazza
}

December 17, 2009

\begin{abstract}
We show that the maximal Nevanlinna counting function and the Carleson function of analytic self-maps of the unit disk are equivalent, up to constants.
\end{abstract}

Mathematics Subject Classification. Primary: 30C80 - Secondary: 47B33; 47B10

Key-words. Analytic self-map of the unit disk - Carleson function - Carleson measure - composition operator - Nevanlinna counting function

\section{Introduction}

Carleson measures and the Nevanlinna counting function are two classical concepts in Complex Analysis. Carleson measures emerged in 1958 when L. Carleson ([1], 2]) showed his famous embedding theorem: For any positive finite measure $\mu$ on the closed unit disk $\overline{\mathbb{D}}$, the identity map from the Hardy space $H^{2}$ into $L^{2}(\mu)$ is bounded if and only if this measure satisfies the following geometric condition: $\sup _{|\xi|=1} \mu[W(\xi, h)]=O(h)$, where $W(\xi, h)$ is the Carleson window of size $h$ centered at $\xi$. This supremum is called the Carleson function $\rho_{\mu}$ of $\mu$.

If $\varphi$ is an analytic self-map of $\mathbb{D}$ (such a function is sometimes called a Schur function), $\varphi$ induces a composition operator $C_{\varphi}: f \in H^{2} \mapsto f \circ \varphi \in H^{2}$, which may be seen as the identity from $H^{2}$ into $L^{2}\left(m_{\varphi}\right)$, where $\mu=m_{\varphi}$ is the image of the Lebesgue measure on the unit circle by $\varphi^{*}$, the boundary values function of $\varphi$. We say that $\rho_{\varphi}=\rho_{m_{\varphi}}$ is the Carleson function of $\varphi$.

Nevanlinna counting function traces back earlier, in the thirties of the last century, in connection with the Jensen formula and the Nevanlinna theory of defect ([18] or [16]). It is defined, for $w \in \varphi(\mathbb{D})$ and $w \neq \varphi(0)$, by $N_{\varphi}(w)=$ $\sum_{\varphi(z)=w} \log 1 /|z|($ see $(2.4))$.

In a slightly different context, Littlewood used it implicitly ([11], see Theorem 4) when he showed that, for every analytic self-map $\varphi$ of $\mathbb{D}$, we have $N_{\varphi}(z)=O(1-|z|)$ as $|z| \rightarrow 1$. This turns out to imply (20], [19]) that the 
composition operator $f \mapsto f \circ \varphi=C_{\varphi}(f)$ is continuous on $H^{2}$ (which precisely means, in present language, that $m_{\varphi}$ is a Carleson measure).

Later, and till now, the regularity of composition operators $C_{\varphi}$ on $H^{2}$ (their compactness, or membership in a Schatten class) in terms of their "symbol" $\varphi$ has been studied either from the point of view of Carleson measures or from the point of view of the Nevanlinna counting function, those two points of view being completely separated. For example, the compactness of $C_{\varphi}: H^{2} \rightarrow H^{2}$ has been characterized in terms of the Carleson function of the symbol $\rho_{\varphi}(h)=o(h)$, as $h \rightarrow 0$, by B. McCluer (14 - see also [17]). In another paper, it was characterized in terms of the Nevanlinna counting function $N_{\varphi}$ of the symbol: $N_{\varphi}(w)=o(1-|w|)$, as $|w| \rightarrow 1$, by J. Shapiro (20|). A similar situation exists for the characterization of the membership of $C_{\varphi}$ in a prescribed Schatten class (12] and [13]).

Though the definition the Carleson measure $m_{\varphi}$ and that of the Nevanlinna counting function $N_{\varphi}$ are of different nature, there should therefore exist a direct link between these two quantities.

Some results in this direction had been given: B. R. Choe (画) showed that $\lim \sup _{h \rightarrow 0}\left(\rho_{\varphi}(h) / h\right)^{1 / 2}$ is equivalent, up to constants, to the distance of $C_{\varphi}$ to the space of compact operators on $H^{2}$; since J. Shapiro proved ([20]) that this distance is $\lim \sup _{|w| \rightarrow 1}\left(N_{\varphi}(w) / \log |w|\right)^{1 / 2}$, one gets that

$$
\limsup _{|w| \rightarrow 1} N_{\varphi}(w) / \log |w| \approx \limsup _{h \rightarrow 0} \rho_{\varphi}(h) / h .
$$

Later, J. S. Choa and H. O. Kim (河) gave a somewhat direct proof of the equivalence of the two above conditions, without using the properties of the composition operator, but without giving explicitly a direct relation between the two functions $\rho_{\varphi}$ and $N_{\varphi}$.

The aim of this paper is to show the surprising fact that the Nevanlinna counting function and the Carleson function are actually equivalent, in the following sense:

Theorem 1.1 There exists a universal constant $C>1$, such that, for every analytic self-map $\varphi: \mathbb{D} \rightarrow \mathbb{D}$, one has:

$$
(1 / C) \rho_{\varphi}(h / C) \leq \sup _{|w| \geq 1-h} N_{\varphi}(w) \leq C \rho_{\varphi}(C h),
$$

for $0<h<1$ small enough.

More precisely, for every $\xi \in \partial \mathbb{D}$, one has:

$$
(1 / 64) m_{\varphi}[W(\xi, h / 64)] \leq \sup _{w \in W(\xi, h) \cap \mathbb{D}} N_{\varphi}(w) \leq 196 m_{\varphi}[W(\xi, 24 h)],
$$

for $0<h<(1-|\varphi(0)|) / 16$. 
Actually the above explicit constants are not relevant and we did not try to have "best" constants. It can be shown that for every $\alpha>1$, there is a constant $C_{\alpha}>0$ such that $m_{\varphi}(S(\xi, h)) \leq C_{\alpha} \tilde{\nu}_{\varphi}(\xi, \alpha h)$ and $\tilde{\nu}_{\varphi}(\xi, h) \leq C_{\alpha} m_{\varphi}(S(\xi, \alpha h))$ for $0<h<(1-|\varphi(0)|) / \alpha$, where $S(\xi, h)$ is defined in (2.3) and $\tilde{\nu}(\xi, h)=$ $\sup _{w \in S(\xi, h) \cap \mathbb{D}} N_{\varphi}(w)$ (see (4.7)).

\section{Notation}

We shall denote by $\mathbb{D}=\{z \in \mathbb{C} ;|z|<1\}$ the open unit disc of the complex plane and by $\mathbb{T}=\partial \mathbb{D}=\{z \in \mathbb{C} ;|z|=1\}$ its boundary; $m$ will be the normalized Lebesgue measure $d t / 2 \pi$ on $\mathbb{T}$, and $A$ the normalized Lebesgue measure $d x d y / \pi$ on $\overline{\mathbb{D}}$. For every analytic self-map $\varphi$ of $\mathbb{D}, m_{\varphi}$ will be the pull-back measure of $m$ by $\varphi^{*}$, where $\varphi^{*}$ is the boundary values function of $\varphi$.

For every $\xi \in \mathbb{T}$ and $0<h<1$, the Carleson window $W(\xi, h)$ centered at $\xi$ and of size $h$ is the set

$$
W(\xi, h)=\{z \in \overline{\mathbb{D}} ;|z| \geq 1-h \quad \text { and } \quad|\arg (z \bar{\xi})| \leq h\} .
$$

For convenience, we shall set $W(\xi, h)=\overline{\mathbb{D}}$ for $h \geq 1$.

For every analytic self-map $\varphi$ of $\mathbb{D}$, one defines the maximal function of $m_{\varphi}$, for $0<h<1$, by:

$$
\rho_{\varphi}(h)=\sup _{\xi \in \mathbb{T}} m\left(\left\{\zeta \in \mathbb{T} ; \varphi^{*}(\zeta) \in W(\xi, h)\right\}\right)=\sup _{\xi \in \mathbb{T}} m_{\varphi}(W(\xi, h)) .
$$

We have $\rho_{\varphi}(h)=1$ for $h \geq 1$. We shall call this function $\rho_{\varphi}$ the Carleson function of $\varphi$. For convenience, we shall often also use, instead of the Carleson window $W(\xi, h)$, the set

$$
S(\xi, h)=\{z \in \overline{\mathbb{D}} ;|z-\xi| \leq h\},
$$

which has an equivalent size.

The Nevanlinna counting function $N_{\varphi}$ is defined, for $w \in \varphi(\mathbb{D}) \backslash\{\varphi(0)\}$, by

$$
N_{\varphi}(w)=\sum_{\varphi(z)=w} \log \frac{1}{|z|}
$$

each term $\log \frac{1}{|z|}$ being repeated according to the multiplicity of $z$, and $N_{\varphi}(w)=$ 0 for the other $w \in \mathbb{D}$. Its maximal function will be denoted by

$$
\nu_{\varphi}(t)=\sup _{|w| \geq 1-t} N_{\varphi}(w) .
$$

\section{Majorizing the Nevanlinna counting function by the Carleson function}

The goal of this section is to prove: 
Theorem 3.1 For every analytic self-map $\varphi$ of $\mathbb{D}$, one has, for every $a \in \mathbb{D}$ :

$$
N_{\varphi}(a) \leq 196 m_{\varphi}(W(\xi, 12 h)),
$$

for $0<h<(1-|\varphi(0)|) / 4$, where $\xi=\frac{a}{|a|}$ and $h=1-|a|$.

In particular, for $0<h<(1-|\varphi(0)|) / 4$ :

$$
\nu_{\varphi}(h)=\sup _{|a| \geq 1-h} N_{\varphi}(a) \leq 196 \rho_{\varphi}(12 h) .
$$

Let us note that, since $W(\zeta, s) \subseteq W(\xi, 2 t)$ whenever $0<s \leq t$ and $\zeta \in$ $W(\xi, t) \cap \partial \mathbb{D}$, we get from (3.1) that

$$
\sup _{w \in W(\xi, h) \cap \mathbb{D}} N_{\varphi}(w) \leq 196 m_{\varphi}(W(\xi, 24 h)) .
$$

We shall first prove the following lemma.

Lemma 3.2 Let $\varphi$ be an analytic self map of $\mathbb{D}$. For every $z \in \mathbb{D}$, one has, if $w=\varphi(z), \xi=w /|w|$ and $h=1-|w| \leq 1 / 4$ :

$$
m_{\varphi}(W(\xi, 12 h)) \geq m_{\varphi}(S(\xi, 6 h)) \geq \frac{|w|}{8}(1-|z|) .
$$

Proof. We may assume, by making a rotation, that $w$ is real and positive: $3 / 4 \leq w<1$.

Let:

$$
T(u)=\frac{a u+1}{u+a}
$$

where

$$
a=w-\frac{2}{w}<-1,
$$

so that $T: \mathbb{D} \rightarrow \mathbb{D}$ is analytic, and $T(w)=w / 2$.

If $P_{z}$ is the Poisson kernel at $z$, one has:

$$
\frac{w}{2}=T[\varphi(z)]=\int_{\mathbb{T}}(T \circ \varphi)^{*} P_{z} d m=\int_{\mathbb{T}} \mathfrak{R e}\left[(T \circ \varphi)^{*}\right] P_{z} d m .
$$

Hence, if one sets:

$$
E=\left\{\mathfrak{R e}\left(T \circ \varphi^{*}\right) \geq w / 4\right\}=\left\{\mathfrak{R e}\left[(T \circ \varphi)^{*}\right] \geq w / 4\right\},
$$

one has:

$$
\frac{w}{2} \leq \int_{E} P_{z} d m+\frac{w}{4} \int_{E^{c}} P_{z} d m \leq \int_{E} P_{z} d m+\frac{w}{4} \int_{\mathbb{D}} P_{z} d m=\int_{E} P_{z} d m+\frac{w}{4}
$$

therefore:

$$
\int_{E} P_{z} d m \geq \frac{w}{4}
$$


Since

$$
\left\|P_{z}\right\|_{\infty}=\frac{1+|z|}{1-|z|} \leq \frac{2}{1-|z|}
$$

we get:

$$
m(E) \geq \frac{w}{8}(1-|z|) .
$$

On the other hand, (3.5) writes

$$
u=T^{-1}(U)=\frac{a U-1}{a-U} ;
$$

hence:

$$
|1-u|=|a+1| \frac{|1-U|}{|a-U|} \leq \frac{2|a+1|}{|a-U|} .
$$

But $a<-1$ is negative, so $\mathfrak{R e} U \geq w / 4$ implies that

$$
|a-U| \geq \mathfrak{R e}(U-a) \geq \frac{w}{4}-a=\frac{2}{w}-\frac{3}{4} w \geq \frac{5}{4} .
$$

Moreover, for $w \geq 3 / 4$ :

$$
|a+1|=(1-w)\left(\frac{2}{w}+1\right) \leq \frac{11}{3}(1-w) .
$$

We get hence $|1-u| \leq 6 h$ when (3.7) holds and $\mathfrak{R e} U \geq w / 4$.

It follows that:

$$
\varphi^{*}(E) \subseteq T^{-1}(\{\Re \mathrm{Re} U \geq w / 4\}) \subseteq S(1,6 h),
$$

giving $m_{\varphi}(W(1,12 h)) \geq m_{\varphi}(S(1,6 h)) \geq m(E)$.

Combining this with 3.6 , that finishes the proof.

Remark. Theorem 3.1 follows immediately when $\varphi$ is univalent since then, for $|w| \geq 3 / 4$ and $\varphi(z)=w$ :

$$
N_{\varphi}(w)=\log \frac{1}{|z|} \approx(1-|z|) \lesssim m_{\varphi}(W(1,12 h)) .
$$

When proving the equivalence between the conditions $\rho_{\varphi}(h)=o(h)$, as $h \rightarrow 0$, and $N_{\varphi}(w)=o(1-|w|)$, as $|w| \rightarrow 1$, J. S. Choa and H. O. Kim proved (see [3], page 112) the following inequality, for every analytic self-map $\varphi: \mathbb{D} \rightarrow \mathbb{D}$ and every $w \in \mathbb{D}$, close enough to 1 :

$$
N_{\varphi}(w) \leq \frac{\left(1-|w|^{2}\right)^{2}}{8|w|^{2}} \int_{\partial \mathbb{D}} \frac{1}{|1-\bar{w} \varphi(z)|^{2}} d m(z) .
$$


This result follows from an Hilbertian method, viz. Littlewood-Paley's identity:

$$
\|f \circ \varphi\|_{2}^{2}=|f \circ \varphi(0)|^{2}+2 \int_{\mathbb{D}}\left|f^{\prime}(w)\right|^{2} N_{\varphi}(w) d A(w)
$$

for every $f \in H^{2}$. With (3.9), one cannot go beyond the order 2; for instance, we can deduce from (3.9) (see the proof of Theorem 3.1 below), that, for $0<$ $h \leq 1 / 2$ :

$$
\sup _{|w|=1-h} N_{\varphi}(w) \lesssim h^{2} \int_{0}^{1 / h^{2}} \rho_{\varphi}\left(\frac{1}{\sqrt{t}}\right) d t \lesssim h^{2}+h^{2} \int_{h}^{1} \frac{\rho_{\varphi}(u)}{u^{3}} d u .
$$

This is of course interesting only when the second term in the last sum is at most of order $h^{2}$, so, when the integral is bounded. Nevertheless, this result suffices to show that Shapiro's criterion of compactness for $C_{\varphi}: H^{2} \rightarrow H^{2}$ is implied by McCluer's one. Moreover, when the pull-back measure $m_{\varphi}$ is an $\alpha$-Carleson measure (i.e. $\rho_{\varphi}(h) \leq C h^{\alpha}$ for some constant $C>0$ ), with $1 \leq \alpha \leq 2$, we get

$$
N_{\varphi}(w) \lesssim h^{2}+h^{2} \int_{h}^{1} \frac{u^{\alpha}}{u^{3}} d u \lesssim h^{2}+h^{2} h^{\alpha-2} \lesssim h^{\alpha} .
$$

Recall ([8], Corollary 3.2) that, when $m_{\varphi}$ is an $\alpha$-Carleson measure, the composition operator $C_{\varphi}$ is in the Schatten class $S_{p}$ on the Hardy space $H^{2}$, for every $p>2 /(\alpha-1)$, and that $m_{\varphi}$ is $\alpha$-Carleson for every $\alpha \geq 1$ when $C_{\varphi}: H^{\Psi} \rightarrow H^{\Psi}$ is compact, if $\Psi$ is an Orlicz function satisfying the growth condition $\Delta^{2}$ ([9], Theorem 5.2).

But (3.11) does not suffice for the compactness of $C_{\varphi}: H^{\Psi} \rightarrow H^{\Psi}$ on general Hardy-Orlicz spaces (see [7] or [6]).

In order to prove Theorem 3.1, we shall replace the Littlewood-Paley identity, by a more general formula, deduced from Stanton's formula (see 5 , Theorem 2).

Theorem 3.3 (Stanton's formula) For every analytic self-map $\varphi: \mathbb{D} \rightarrow \mathbb{D}$ and every subharmonic function $G: \mathbb{D} \rightarrow \mathbb{R}$, one has:

$$
\lim _{r \uparrow 1} \int_{\partial \mathbb{D}} G[\varphi(r \xi)] d m(\xi)=G[\varphi(0)]+\frac{1}{2} \int_{\mathbb{D}} \Delta G(w) N_{\varphi}(w) d A(w),
$$

where $\Delta$ is the distributional Laplacian.

Proof of Theorem 3.1. If $a \notin \varphi(\mathbb{D})$, one has $N_{\varphi}(a)=0$, and the result is trivial. We shall hence assume that $a \in \varphi(\mathbb{D})$.

Let $\Phi:[0, \infty) \rightarrow[0, \infty)$ be an Orlicz function, that is a non-decreasing convex function such that $\Phi(0)=0$ and $\Phi(\infty)=\infty$, and we assume that $\Phi^{\prime}$ is also an Orlicz function. In other words, $\Phi^{\prime \prime}$ is an arbitrary non-negative and nondecreasing function and $\Phi^{\prime}(x)=\int_{0}^{x} \Phi^{\prime \prime}(t) d t$ and $\Phi(x)=\int_{0}^{x} \Phi^{\prime}(t) d t$. 
Let now $f: \mathbb{D} \rightarrow \mathbb{C}$ be an analytic function. We have, outside the zeroes of $f$, in writing $\Delta \Phi(|f|)=4 \partial \bar{\partial} \Phi\left(\sqrt{|f|^{2}}\right)$ :

$$
\Delta \Phi(|f|)=\left[\Phi^{\prime \prime}(|f|)+\frac{\Phi^{\prime}(|f|)}{|f|}\right]\left|f^{\prime}\right|^{2} .
$$

We shall only use here that:

$$
\Delta \Phi(|f|) \geq \Phi^{\prime \prime}(|f|)\left|f^{\prime}\right|^{2}
$$

(this is a not too crude estimate, since, $\Phi^{\prime}$ being an Orlicz function, $\Phi^{\prime \prime}$ is non-negative and non-decreasing, and hence $\Phi^{\prime}(x)=\int_{0}^{x} \Phi^{\prime \prime}(t) d t \leq x \Phi^{\prime \prime}(x)$ and $\left.\Phi^{\prime}(x)=\int_{0}^{x} \Phi^{\prime \prime}(t) d t \geq \int_{x / 2}^{x} \Phi^{\prime \prime}(t) d t \geq(x / 2) \Phi^{\prime \prime}(x / 2)\right)$.

Set now, for $a \in \mathbb{D}$ :

$$
f_{a}(z)=\frac{1-|a|}{1-\bar{a} z}, \quad z \in \overline{\mathbb{D}} .
$$

Since $\Phi\left(\left|f_{a}\right|\right)$ is subharmonic ( $\Phi$ being convex and non-decreasing) and bounded, we can use Stanton's formula as:

$$
\int_{\partial \mathbb{D}} \Phi\left(\left|f_{a} \circ \varphi\right|\right) d m \geq \frac{1}{2} \int_{\mathbb{D}} \Phi^{\prime \prime}\left(\left|f_{a}\right|\right)\left|f_{a}^{\prime}\right|^{2} N_{\varphi} d A .
$$

Let $h=1-|a|$. For $|z-a|<h$, one has

$$
|1-\bar{a} z|=\left|\left(1-|a|^{2}\right)+\bar{a}(a-z)\right| \leq\left(1-|a|^{2}\right)+|a-z| \leq 2 h+h=3 h ;
$$

Hence $\left|f_{a}(z)\right| \geq \frac{h}{3 h}=\frac{1}{3}$ for $|z-a|<h$. It follows, since $\Phi^{\prime \prime}$ is non-decreasing:

$$
\int_{\partial \mathbb{D}} \Phi\left(\left|f_{a} \circ \varphi\right|\right) d m \geq \frac{1}{2} \Phi^{\prime \prime}\left(\frac{1}{3}\right) \int_{D(a, h)}\left|f_{a}^{\prime}\right|^{2} N_{\varphi} d A .
$$

Now, if $\varphi_{a}(z)=\frac{a-z}{1-\bar{a} z}$, one has $\left|f_{a}^{\prime}(z)\right|=\frac{|a|}{1+|a|}\left|\varphi_{a}^{\prime}(z)\right| \geq \frac{3}{7}\left|\varphi_{a}^{\prime}(z)\right|$ (we may, and do, assume that $1-|a|=h \leq 1 / 4)$; hence:

$$
\begin{aligned}
\int_{\partial \mathbb{D}} \Phi\left(\left|f_{a} \circ \varphi\right|\right) d m & \geq \frac{1}{2} \Phi^{\prime \prime}\left(\frac{1}{3}\right) \frac{9}{49} \int_{D(a, h)}\left|\varphi_{a}^{\prime}\right|^{2} N_{\varphi} d A \\
& =\frac{9}{98} \Phi^{\prime \prime}\left(\frac{1}{3}\right) \int_{\varphi_{a}(D(a, h))} N_{\varphi_{a} \circ \varphi} d A
\end{aligned}
$$

(because $N_{\varphi_{a} \circ \varphi}\left(\varphi_{a}(w)\right)=N_{\varphi}(w)$ and $\left.\varphi_{a}^{-1}=\varphi_{a}\right)$.

But $\varphi_{a}(D(a, h)) \supseteq D(0,1 / 3)$ : indeed, if $|w|<1 / 3$, then $w=\varphi_{a}(z)$, with

$$
|a-z|=\left|\frac{\left(1-|a|^{2}\right) w}{1-\bar{a} w}\right| \leq\left(1-|a|^{2}\right) \frac{|w|}{1-|w|}<2 h \frac{1 / 3}{1-1 / 3}=h .
$$


We are going now to use the sub-averaging property of the Nevanlinna function (19], page 190, 20], § 4.6, or [21], Proposition 10.2.4): for every analytic self-map $\psi: \mathbb{D} \rightarrow \mathbb{D}$, one has

$$
N_{\psi}\left(w_{0}\right) \leq \frac{1}{A(\Delta)} \int_{\Delta} N_{\psi}(w) d A(w),
$$

for every disk $\Delta$ of center $w_{0}$ which does not contain $\psi(0)$.

This will be possible thanks to the following:

Lemma 3.4 For $1-|a|<(1-|\varphi(0)|) / 4$, one has $\left|\left(\varphi_{a} \circ \varphi\right)(0)\right|>1 / 3$.

Proof. One has $|1-\bar{a} \varphi(0)| \leq\left(1-|a|^{2}\right)+|\bar{a}||a-\varphi(0)| \leq\left(1-|a|^{2}\right)+|a-\varphi(0)| ;$ hence:

$$
\begin{aligned}
\left|\varphi_{a}(\varphi(0))\right| \geq \frac{|a-\varphi(0)|}{\left(1-|a|^{2}\right)+|a-\varphi(0)|} & \geq 1-\frac{1-|a|^{2}}{\left(1-|a|^{2}\right)+|a-\varphi(0)|} \\
& \geq 1-\frac{1-|a|^{2}}{|a-\varphi(0)|} \geq 1-2 \frac{1-|a|}{|a-\varphi(0)|} .
\end{aligned}
$$

But when $1-|a|<(1-|\varphi(0)|) / 4$, one has:

$$
|a-\varphi(0)| \geq|a|-|\varphi(0)|=(1-|\varphi(0)|)-(1-|a|)>3(1-|a|),
$$

and the result follows.

Hence:

$$
\int_{D(0,1 / 3)} N_{\varphi_{a} \circ \varphi} d A \geq \frac{1}{9} N_{\varphi_{a} \circ \varphi}(0)=\frac{1}{9} N_{\varphi}(a),
$$

and

$$
\int_{\partial \mathbb{D}} \Phi\left(\left|f_{a} \circ \varphi\right|\right) d m \geq \frac{1}{98} \Phi^{\prime \prime}\left(\frac{1}{3}\right) N_{\varphi}(a) .
$$

We now have to estimate from above $\int_{\partial \mathbb{D}} \Phi\left(\left|f_{a} \circ \varphi\right|\right) d m$. For that, we shall use the following easy lemma.

Lemma 3.5 For every $\xi \in \partial \mathbb{D}$ and every $h \in(0,1 / 2]$, one has:

$$
|1-\bar{a} z|^{2} \geq \frac{1}{4}\left(h^{2}+|z-\xi|^{2}\right), \quad \forall z \in \overline{\mathbb{D}},
$$

where $a=(1-h) \xi$.

Proof. The result is rotation-invariant; so we may assume that $\xi=1$ (and hence $a>0$ ). Write $z=1-r \mathrm{e}^{i \theta}$. Since $|z| \leq 1$ if and only if $r \leq 2 \cos \theta$, one has $\cos \theta \geq 0$ and hence $|\theta| \leq \pi / 2$. Then:

$$
\begin{aligned}
|1-\bar{a} z|^{2} & =\left|1-a\left(1-r \mathrm{e}^{i \theta}\right)\right|^{2}=\left|1-a+a r \mathrm{e}^{i \theta}\right|^{2} \\
& =(1-a)^{2}+a^{2} r^{2}+2 a r(1-a) \cos \theta \\
& \geq(1-a)^{2}+a^{2} r^{2} \geq \frac{1}{4}\left(h^{2}+r^{2}\right)=\frac{1}{4}\left(h^{2}+|z-1|^{2}\right) .
\end{aligned}
$$


Then:

$$
\begin{aligned}
\int_{\partial \mathbb{D}} \Phi\left(\left|f_{a} \circ \varphi\right|\right) d m & =\int_{\overline{\mathbb{D}}} \Phi\left(\frac{1-|a|}{|1-\bar{a} z|}\right) d m_{\varphi}(z) \\
& \leq \int_{\overline{\mathbb{D}}} \Phi\left(\frac{2 h}{\left(h^{2}+|z-\xi|^{2}\right)^{1 / 2}}\right) d m_{\varphi}(z), \quad \text { by }(3.19) \\
& =\int_{0}^{+\infty} m_{\varphi}\left(\Phi\left(\frac{2 h}{\left(h^{2}+|z-\xi|^{2}\right)^{1 / 2}}\right) \geq t\right) d t \\
& =\int_{0}^{+\infty} m_{\varphi}\left(\left(h^{2}+|z-\xi|^{2}\right)^{1 / 2} \leq 2 h / \Phi^{-1}(t)\right) d t \\
& =\int_{0}^{\Phi(2)} m_{\varphi}\left(\left(h^{2}+|z-\xi|^{2}\right)^{1 / 2} \leq 2 h / \Phi^{-1}(t)\right) d t
\end{aligned}
$$

since $h \leq\left(h^{2}+|z-\xi|^{2}\right)^{1 / 2} \leq 2 h / \Phi^{-1}(t)$ implies $t \leq \Phi(2)$. We get:

$$
\int_{\partial \mathbb{D}} \Phi\left(\left|f_{a} \circ \varphi\right|\right) d m \leq \int_{0}^{\Phi(2)} m_{\varphi}\left(|z-\xi| \leq 2 h / \Phi^{-1}(t)\right) d t .
$$

We obtain from 3.18 , by setting $u=2 h / \Phi^{-1}(t)$ :

$$
N_{\varphi}(a) \leq \frac{98}{\Phi^{\prime \prime}(1 / 3)} \int_{h}^{\infty} m_{\varphi}(S(\xi, u)) \frac{2 h}{u^{2}} \Phi^{\prime}\left(\frac{2 h}{u}\right) d u .
$$

Since $\Phi^{\prime}(x) \leq x \Phi^{\prime \prime}(x)$, we get:

$$
N_{\varphi}(a) \leq \frac{98}{\Phi^{\prime \prime}(1 / 3)} \int_{h}^{\infty} m_{\varphi}(S(\xi, u)) \frac{4 h^{2}}{u^{3}} \Phi^{\prime \prime}\left(\frac{2 h}{u}\right) d u .
$$

We are going now to choose suitably the Orlicz function $\Phi$. It suffices to define $\Phi^{\prime \prime}$, for $a \in \mathbb{D}$ given (with $\xi=a /|a|$ and $h=1-|a| \leq 1 / 4$ ). By Lemma 3.2, since $a \in \varphi(\mathbb{D})$, there is a constant $c_{0}>0$, such that $m_{\varphi}\left(S\left(\xi, c_{0} h\right)\right)>0$; we can hence set (note that $m_{\varphi}(S(\xi, u)) \leq 1$ ):

$$
\Phi^{\prime \prime}(v)=\left\{\begin{array}{cl}
1 & \text { if } 0 \leq v \leq h, \\
\frac{1}{m_{\varphi}(S(\xi, 2 h / v))} & \text { if } h \leq v \leq 2 / c_{0}, \\
\frac{1}{m_{\varphi}\left(S\left(\xi, c_{0} h\right)\right)} & \text { if } v \geq 2 / c_{0} .
\end{array}\right.
$$

It is a non-negative non-decreasing function, so the assumptions made on $\Phi$ at the beginning are satisfied. One has, since $m_{\varphi}(S(\xi, u)) \Phi^{\prime \prime}(2 h / u) \leq 1$ :

$$
\int_{h}^{\infty} m_{\varphi}(S(\xi, u)) \frac{4 h^{2}}{u^{3}} \Phi^{\prime \prime}\left(\frac{2 h}{u}\right) d u \leq \int_{h}^{\infty} \frac{4 h^{2}}{u^{3}} d u=2 .
$$

Since $c_{0} \leq 6$, one has $h \leq 1 / 3 \leq 2 / c_{0}$ and hence $\Phi^{\prime \prime}(1 / 3)=1 / m_{\varphi}(S(\xi, 6 h))$; therefore 3.20$)$ gives, for $h \leq(1-|\varphi(0)|) / 4$ :

$$
N_{\varphi}(a) \leq 196 m_{\varphi}(S(\xi, 6 h))
$$

finishing the proof since $S(\xi, 6 h) \subseteq W(\xi, 12 h)$. 


\section{Domination of the Carleson function by the Nevanlinna function}

We cannot expect to estimate individually from above the $m_{\varphi}$-measure of Carleson windows centered at $\xi=w /|w|$ by $N_{\varphi}(w)$, as in Theorem 3.1. In fact, consider a conformal mapping $\varphi$ from $\mathbb{D}$ onto $\mathbb{D} \backslash\left[0,1\left[\right.\right.$. One has $N_{\varphi}(t)=0$ for every $t \in\left[0,1\left[\right.\right.$, though $m_{\varphi}(W(1, h))>0$ for every $h>0$ (because $W(1, h) \supset$ $W\left(\mathrm{e}^{i h / 2}, h / 2\right)$ and $m_{\varphi}\left(W\left(\mathrm{e}^{i h / 2}, h / 2\right)\right)>0$ by Lemma 3.2).

Let us give another example. Let $\varphi(z)=(1+z) / 2$. Then:

a) One has $\varphi\left(\mathrm{e}^{i \theta}\right)=(\cos \theta / 2) \mathrm{e}^{i \theta / 2}($ with $|\theta| \leq \pi)$. Hence $\varphi\left(\mathrm{e}^{i \theta}\right) \in W\left(\mathrm{e}^{i \theta_{0}}, h\right)$ if and only if $\cos (\theta / 2) \geq 1-h$ and $\left|(\theta / 2)-\theta_{0}\right| \leq h$, i.e. $2\left(\theta_{0}-h\right) \leq \theta \leq 2\left(\theta_{0}+h\right)$.

Now, $1-\cos (\theta / 2) \leq \theta^{2} / 8$, so the modulus condition is satisfied when $\theta^{2} \leq 8 h$; in particular when $|\theta| \leq 2 \sqrt{h}$.

For $\theta_{0}=\sqrt{h}, m_{\varphi}\left(W\left(\mathrm{e}^{i \theta_{0}}, h\right)\right)$ is bigger than the length of the interval

$$
[-2 \sqrt{h}, 2 \sqrt{h}] \cap[2(\sqrt{h}-h), 2(\sqrt{h}+h)]=[2 \sqrt{h}-2 h, 2 \sqrt{h}],
$$

that is $2 h$. Therefore $m_{\varphi}\left(W\left(\mathrm{e}^{i \theta_{0}}, h\right)\right) \geq 2 h$.

b) Let now $w=\varphi(z)$. Write $w=\frac{1}{2}+r \mathrm{e}^{i \zeta}$ with $0 \leq r<1 / 2$. Then, writing $r=\frac{1}{2}-s$, one has $|z|=|2 w-1|=2 r$ and

$$
N_{\varphi}(w)=\log \frac{1}{|z|}=\log \frac{1}{2 r}=\log \frac{1}{1-2 s} \approx s .
$$

Now, $|w|^{2}=\frac{1}{4}+r^{2}+r \cos \zeta$ and

$$
h \approx 1-|w|^{2}=\frac{1}{2}(1-\cos \zeta)+s(1+\cos \zeta)-s^{2} \approx \frac{\zeta^{2}}{4}+2 s .
$$

Writing $\zeta=s^{1 / 2 \alpha}$, one gets:

(i) for "small" $\zeta($ i.e. $0<\alpha \leq 1): h \approx s$, and so $N_{\varphi}(w) \approx h$;

(ii) for "large" (i.e. $\alpha \geq 1$ ): $h \approx s^{1 / \alpha}$, and so $N_{\varphi}(w) \approx h^{\alpha}$.

On the other hand, $w=\mathrm{e}^{i \zeta / 2}[(1-s) \cos (\zeta / 2)-i s \sin (\zeta / 2)]$; hence, when $s$ goes to 0 , one has

$$
\theta_{w}:=\arg w=\frac{\zeta}{2}+\arctan \left[\frac{s \sin (\zeta / 2)}{(1-s) \cos (\zeta / 2)}\right] \sim \frac{\zeta}{2} \approx \zeta .
$$

For $\alpha \geq 1$, one has $h \approx s^{1 / \alpha}=\zeta^{2}$, i.e. $\zeta \approx \sqrt{h}$. Then, choosing $\alpha>1$ such that $\zeta=\theta_{0}$, one has $m_{\varphi}(W(w /|w|, h)) \approx h$, though $N_{\varphi}(w) \approx h^{\alpha} \ll h$.

One cannot hence dominate $m_{\varphi}(W(w /|w|, h))$ by $N_{\varphi}(w)$.

We can remark that, nevertheless, in either case, one has $\rho_{\varphi}(h) \approx h$ and $\nu_{\varphi}(h) \approx h$.

We shall prove: 
Theorem 4.1 For every analytic self-map $\varphi: \mathbb{D} \rightarrow \mathbb{D}$, one has, for every $\xi \in$ $\partial \mathbb{D}$ :

$$
m_{\varphi}(W(\xi, h)) \leq 64 \sup _{w \in W(\xi, 64 h) \cap \mathbb{D}} N_{\varphi}(w),
$$

for $0<h<(1-|\varphi(0)|) / 16$.

Proof. We shall set:

$$
\nu_{\varphi}(\xi, h)=\sup _{w \in W(\xi, h) \cap \mathbb{D}} N_{\varphi}(w) .
$$

Note that

$$
\nu_{\varphi}(h)=\sup _{|\xi|=1} \nu_{\varphi}(\xi, h),
$$

where $\nu_{\varphi}$ is defined in (2.5)

If for some $h_{0}>0$, one has $\nu_{\varphi}\left(\xi, h_{0}\right)=0$, then $\varphi(\mathbb{D}) \subseteq \mathbb{D} \backslash W\left(\xi, h_{0}\right)$, and hence $m_{\varphi}(W(\xi, h))=0$ for $0<h<h_{0}$. Therefore we shall assume that $\nu_{\varphi}(\xi, h)>0$. We may, and do, also assume that $h \leq 1 / 4$. By replacing $\varphi$ by $\mathrm{e}^{i \theta} \varphi$, it suffices to estimate $m_{\varphi}(S(1, h)$ ) (recall that $S(1, t)=\{z \in \overline{\mathbb{D}} ;|1-z| \leq t\}$ ).

We shall use the same functions $f_{a}$ as in the proof of Theorem 3.1, but, for convenience, with a different notation. We set, for $0<r<1$ :

$$
u(z)=\frac{1-r}{1-r z} .
$$

Let us take an Orlicz function $\Phi$ as in the beginning of the proof of Theorem 3.1, which will be precised later. We shall take this function in such a way that $\Phi(|u(\varphi(0))|)=0$.

Since $\Phi^{\prime}(x) \leq x \Phi^{\prime \prime}(x),(3.13)$ becomes:

$$
\Delta \Phi(|u|) \leq 2 \Phi^{\prime \prime}(|u|)\left|u^{\prime}\right|^{2},
$$

and Stanton's formula writes, since $\Phi(|u(\varphi(0))|)=0$ :

$$
\int_{\partial \mathbb{D}} \Phi(|u \circ \varphi|) d m \leq \int_{\mathbb{D}} \Phi^{\prime \prime}(|u(w)|)\left|u^{\prime}(w)\right|^{2} N_{\varphi}(w) d A(w) .
$$

In all the sequel, we shall fix $h, 0<h \leq 1 / 4$, and take $r=1-h$. so:

For $|z| \leq 1$ and $|1-z| \leq h$, one has $|1-r z|=|(1-z)+h z| \leq|1-z|+h \leq 2 h$,

$$
|u(z)| \geq \frac{(1-r)}{2 h}=\frac{1}{2} .
$$

Hence:

$$
\begin{aligned}
m_{\varphi}(S(1, h)) & \leq \frac{1}{\Phi(1 / 2)} \int_{S(1, h)} \Phi(|u(z)|) d m_{\varphi}(z) \\
& \leq \frac{1}{\Phi(1 / 2)} \int_{\mathbb{D}} \Phi(|u(z)|) d m_{\varphi}(z) \\
& =\frac{1}{\Phi(1 / 2)} \int_{\mathbb{T}} \Phi(|(u \circ \varphi)(z)|) d m(z),
\end{aligned}
$$


and so, by 4.5 :

$$
m_{\varphi}(S(1, h)) \leq \frac{1}{\Phi(1 / 2)} \int_{\mathbb{D}} \Phi^{\prime \prime}(|u(z)|)\left|u^{\prime}(z)\right|^{2} N_{\varphi}(z) d A(z) .
$$

We are going to estimate this integral by separating two cases: $|1-z| \leq h$ and $|1-z|>h$.

For convenience, we shall set:

$$
\tilde{\nu}(t)=\sup _{w \in S(1, t) \cap \mathbb{D}} N_{\varphi}(w) .
$$

1) Remark first that

$$
u^{\prime}(z)=\frac{r h}{(1-r z)^{2}}
$$

and so:

$$
\left|u^{\prime}(z)\right| \leq \frac{h}{(1-r)^{2}}=\frac{1}{h} .
$$

Since $|u(z)| \leq 1$, we get hence:

$$
\int_{|1-z| \leq h} \Phi^{\prime \prime}(|u(z)|)\left|u^{\prime}(z)\right|^{2} N_{\varphi}(z) d A(z) \leq \int_{S(1, h)} \Phi^{\prime \prime}(1) \frac{1}{h^{2}} \tilde{\nu}(h) d A(z),
$$

giving, since $A(S(1, h)) \leq h^{2}$ :

$$
\int_{|1-z| \leq h} \Phi^{\prime \prime}(|u(z)|)\left|u^{\prime}(z)\right|^{2} N_{\varphi}(z) d A(z) \leq \Phi^{\prime \prime}(1) \tilde{\nu}(h) .
$$

2) For $0<h \leq 1 / 4$, one has:

$$
|u(z)| \leq \frac{2 h}{|1-z|} \quad \text { and } \quad\left|u^{\prime}(z)\right| \leq \frac{2 h}{|1-z|^{2}}
$$

indeed, we have (this is obvious, by drawing a picture):

$$
|1-r z|=r\left|\frac{1}{r}-z\right| \geq r|1-z|,
$$

and hence $|1-r z| \geq \frac{3}{4}|1-z|$, since $r=1-h \geq 3 / 4$. We obtain:

$$
\begin{aligned}
& \int_{|1-z|>h} \Phi^{\prime \prime}(|u(z)|)\left|u^{\prime}(z)\right|^{2} N_{\varphi}(z) d A(z) \\
& \leq 4 \int_{|1-z|>h} \Phi^{\prime \prime}\left(\frac{2 h}{|1-z|}\right) \frac{h^{2}}{|1-z|^{4}} N_{\varphi}(z) d A(z) .
\end{aligned}
$$

Then, using polar coordinates centered at 1 (note that we only have to integrate over an arc of length less than $\pi$ ), and the obvious inequality $N_{\varphi}(z) \leq$ 
$\tilde{\nu}(|1-z|)$, we get:

$$
\begin{aligned}
\int_{|1-z|>h} \Phi^{\prime \prime}(|u(z)|)\left|u^{\prime}(z)\right|^{2} & N_{\varphi}(z) d A(z) \\
& \leq 4 \int_{h}^{2} \Phi^{\prime \prime}\left(\frac{2 h}{t}\right) \frac{h^{2}}{t^{3}} \tilde{\nu}(t) d t .
\end{aligned}
$$

We now choose the Orlicz function as follows (with $a=\varphi(0)$ ):

$$
\Phi^{\prime \prime}(v)=\left\{\begin{array}{cl}
0 & \text { if } 0 \leq v \leq h /(1-|a|), \\
\frac{1}{\tilde{\nu}(2 h / v)} & \text { if } h /(1-|a|)<v<2, \\
\frac{1}{\tilde{\nu}(h)} & \text { if } v \geq 2 .
\end{array}\right.
$$

This function is non-negative and non-decreasing. Moreover, one has $\Phi(x)=0$ for $0 \leq x \leq h /(1-|a|)$. Hence, since $|u(a)| \leq \frac{h}{1-|a|}$, one has $\Phi(|u(a)|)=0$.

Then

$$
\begin{aligned}
\int_{h}^{2} \Phi^{\prime \prime}\left(\frac{2 h}{t}\right) \frac{h^{2}}{t^{3}} \tilde{\nu}(t) d t & =\int_{h}^{2(1-|a|)} \Phi^{\prime \prime}\left(\frac{2 h}{t}\right) \frac{h^{2}}{t^{3}} \tilde{\nu}(t) d t \\
& \leq \int_{h}^{\infty} \frac{h^{2}}{t^{3}} d t=\frac{1}{2}
\end{aligned}
$$

Now,

$$
\begin{aligned}
\Phi\left(\frac{1}{2}\right) & =\int_{0}^{1 / 2} \Phi^{\prime}(t) d t \geq \int_{1 / 4}^{1 / 2} \Phi^{\prime}(t) d t \geq \int_{1 / 4}^{1 / 2} \frac{t}{2} \Phi^{\prime \prime}\left(\frac{t}{2}\right) d t \\
& \geq \Phi^{\prime \prime}\left(\frac{1}{8}\right) \int_{1 / 4}^{1 / 2} \frac{t}{2} d t=\frac{3}{64} \Phi^{\prime \prime}\left(\frac{1}{8}\right) .
\end{aligned}
$$

When $h<(1-|a|) / 8$, one has $1 / 8>h /(1-|a|)$; hence $\Phi^{\prime \prime}(1 / 8)=1 / \tilde{\nu}(16 h)$, and $\Phi^{\prime \prime}(1)=1 / \tilde{\nu}(2 h)$. We get hence, from (4.6), (4.8), 4.9) and (4.11):

$$
m_{\varphi}(S(1, h)) \leq \frac{64}{3} \tilde{\nu}(16 h)\left[\frac{\tilde{\nu}(h)}{\tilde{\nu}(2 h)}+2\right] \leq 64 \tilde{\nu}(16 h) .
$$

Since $W(1, t) \subseteq S(1,2 t)$, we get $m_{\varphi}(W(1, h)) \leq 64 \sup _{w \in S(1,32 h)} N_{\varphi}(w)$ for $0<h<(1-|\varphi(0)|) / 16$, and that ends the proof of Theorem 4.1, since $S(1,32 h) \subseteq W(1,64 h)$.

Remark. A slight modification of the proof gives the following improvement, if one allows a (much) bigger constant. 
Theorem 4.2 There are universal constants $C, c>1$ such that

$$
m_{\varphi}(S(\xi, h)) \leq C \frac{1}{A(S(\xi, c h))} \int_{S(\xi, c h)} N_{\varphi}(z) d A(z)
$$

for every analytic self-map $\varphi: \mathbb{D} \rightarrow \mathbb{D}$, every $\xi \in \partial \mathbb{D}$, and $0<h<(1-|\varphi(0)|) / 8$.

Proof. We are going to follow the proof of Theorem 4.1. We shall assume that $\xi=1$ and we set:

$$
I(t)=\int_{S(1, t)} N_{\varphi}(z) d A(z)
$$

Then:

1) When $|1-z|<h$, we have, instead of (4.8):

$$
\begin{aligned}
\int_{|1-z|<h} \Phi^{\prime \prime}(|u(z)|)\left|u^{\prime}(z)\right|^{2} N_{\varphi}(z) d A(z) & \leq \int_{S(1, h)} \Phi^{\prime \prime}(1) \frac{1}{h^{2}} N_{\varphi}(z) d A(z) \\
& =\Phi^{\prime \prime}(1) \frac{1}{h^{2}} I(h)
\end{aligned}
$$

2) For $|z-1| \geq h$, we write:

$$
\begin{aligned}
\int_{|1-z| \geq h} \Phi^{\prime \prime}(|u(z)|)\left|u^{\prime}(z)\right|^{2} & N_{\varphi}(z) d A(z) \\
& =\sum_{k=1}^{\infty} \int_{k h \leq|1-z|<(k+1) h} \Phi^{\prime \prime}(|u(z)|)\left|u^{\prime}(z)\right|^{2} N_{\varphi}(z) d A(z) \\
& \leq 4 \sum_{k=1}^{\infty} \Phi^{\prime \prime}\left(\frac{2 h}{k h}\right) \frac{h^{2}}{k^{4} h^{4}} I((k+1) h) \\
& =4 \sum_{k=1}^{\infty} \Phi^{\prime \prime}\left(\frac{2}{k}\right) \frac{1}{k^{4} h^{2}} I((k+1) h)
\end{aligned}
$$

We take, with $a=\varphi(0)$ :

$$
\Phi^{\prime \prime}(v)=\left\{\begin{array}{cl}
0 & \text { if } 0 \leq v \leq h /(1-|a|) \\
\frac{1}{\left.I\left(\left(\frac{2}{v}+1\right) h\right)\right)} & \text { if } v>h /(1-|a|)
\end{array}\right.
$$

Then

$$
\int_{|1-z| \geq h} \Phi^{\prime \prime}(|u(z)|)\left|u^{\prime}(z)\right|^{2} N_{\varphi}(z) d A(z) \leq \frac{4}{h^{2}} \sum_{k=1}^{\infty} \frac{1}{k^{4}}=\frac{4}{h^{2}} \frac{\pi^{4}}{90} \leq \frac{5}{h^{2}}
$$

Since $h<(1-|a|) / 8$, one has $1 / 8>h /(1-|a|)$; hence $\Phi^{\prime \prime}(1 / 8)=\frac{1}{I(17 h)}$ and 
$\Phi^{\prime \prime}(1)=\frac{1}{I(3 h)}$. Therefore:

$$
\begin{aligned}
m_{\varphi}(S(1, h)) & \leq \frac{64}{3} I(17 h)\left[\frac{1}{h^{2}} \frac{I(h)}{I(3 h)}+\frac{5}{h^{2}}\right] \\
& \leq \frac{64}{3} I(17 h) \frac{6}{h^{2}}=128 \frac{I(17 h)}{h^{2}} \\
& \leq 128 \times 17^{2} \frac{I(17 h)}{A(S(1,17 h))},
\end{aligned}
$$

ending the proof of Theorem 1.2 .

\section{Some consequences}

In [7] (see also [6], Théorème 4.2), we proved (Theorem 4.19) that the Carleson function of an analytic self-map $\varphi$ has the following property of homogeneity, improving that $m_{\varphi}$ is a Carleson measure: $m_{\varphi}(S(\xi, \varepsilon h)) \leq K \varepsilon m_{\varphi}(S(\xi, h))$ for $0<h<1-|\varphi(0)|, 0<\varepsilon<1$ and $\xi \in \partial \mathbb{D}$, where $K$ is a universal constant. It follows from Theorem 1.1. (actually Theorem 3.1 and Theorem 4.1) that:

Theorem 5.1 There exist a universal constant $K>0$ such that, for every analytic self-map $\varphi$ of $\mathbb{D}$, one has, for $0<\varepsilon<1$ :

$$
\nu_{\varphi}(\varepsilon t) \leq K \varepsilon \nu_{\varphi}(t),
$$

for $t$ small enough.

More precisely, for $t$ small enough, one has, for every $\xi \in \partial \mathbb{D}$ :

$$
\nu_{\varphi}(\xi, \varepsilon t) \leq K \varepsilon \nu_{\varphi}(\xi, t),
$$

where $\nu_{\varphi}(\xi, s)=\sup _{w \in W(\xi, s) \cap \mathbb{D}} N_{\varphi}(w)$.

Note that the two above quoted theorems give Theorem 5.1 a priori only for $0<\varepsilon<1 / K$; but if $1 / K \leq \varepsilon<1$, one has $\nu_{\varphi}(\xi, \varepsilon t) \leq \nu_{\varphi}(\xi, t) \leq K \varepsilon \nu_{\varphi}(\xi, t)$.

We shall end this paper with a consequences of Theorem 1.1 for composition operators. Recall that if $\Psi$ is an Orlicz function, the Hardy-Orlicz space is the space of functions $f \in H^{1}$ whose boundary values are in the Orlicz space $L^{\Psi}(\partial \mathbb{D}, m)$. We proved in [7], Theorem 4.18 (see also [6], Théorème 4.2) that, if $\frac{\Psi(x)}{x} \underset{x \rightarrow \infty}{\longrightarrow} \infty$, the composition operator $C_{\varphi}: H^{\Psi} \rightarrow H^{\Psi}$ is compact if and only if, for every $A>0$, one has $\rho_{\varphi}(h)=o\left[1 / \Psi\left(A \Psi^{-1}(1 / h)\right)\right]$ when $h$ goes to 0 ; in other words, if and only if

$$
\lim _{h \rightarrow 0} \frac{\Psi^{-1}(1 / h)}{\Psi^{-1}\left(1 / \rho_{\varphi}(h)\right)}=0 .
$$

This remains true when $H^{\Psi}=H^{1}$. Hence Theorem 1.1 gives: 
Theorem 5.2 Let $\varphi: \mathbb{D} \rightarrow \mathbb{D}$ be an analytic self-map and $\Psi$ be an Orlicz function. Then the composition operator $C_{\varphi}: H^{\Psi} \rightarrow H^{\Psi}$ is compact if and only if

$$
\sup _{|w| \geq 1-h} N_{\varphi}(w)=o\left(\frac{1}{\Psi\left(A \Psi^{-1}(1 / h)\right)}\right), \quad \text { as } h \rightarrow 0, \quad \forall A>0 .
$$

It should be noted, due to the arbitrary $A>0$, that (5.3) may be replaced by

$$
\sup _{|w| \geq 1-h} N_{\varphi}(w) \leq \frac{1}{\Psi\left(A \Psi^{-1}(1 / h)\right)}, \quad \forall A>0,
$$

for $h \leq h_{A}$, and this condition also writes, setting $\nu_{\varphi}(h)=\sup _{|w| \geq 1-h} N_{\varphi}(w)$ $($ see $(2.5))$ :

$$
\lim _{h \rightarrow 0} \frac{\Psi^{-1}(1 / h)}{\Psi^{-1}\left(1 / \nu_{\varphi}(h)\right)}=0
$$

It is known that if $C_{\varphi}: H^{2} \rightarrow H^{2}$ is compact, then $\lim _{|z| \rightarrow 1} \frac{1-|\varphi(z)|}{1-|z|}=\infty$, and that this condition is sufficient when $\varphi$ is univalent, or finitely-valent, but not sufficient in general (see [15] and [19], § 3.2). It follows from Theorem 5.2 that an analogous result holds for Hardy-Orlicz spaces:

Theorem 5.3 Let $\varphi: \mathbb{D} \rightarrow \mathbb{D}$ be an analytic self-map, and $\Psi$ be an Orlicz function. Assume that the composition operator $C_{\varphi}: H^{\Psi} \rightarrow H^{\Psi}$ is compact. Then:

$$
\lim _{|z| \rightarrow 1} \frac{\Psi^{-1}\left(\frac{1}{1-|z|}\right)}{\Psi^{-1}\left(\frac{1}{1-|\varphi(z)|}\right)}=\infty .
$$

Conversely, if $\varphi$ is finitely-valent, then (5.6) suffices for $C_{\varphi}: H^{\Psi} \rightarrow H^{\Psi}$ to be compact.

Recall that the assumption " $\varphi$ is finitely-valent" means that there is an integer $p \geq 1$ such that each $w \in \varphi(\mathbb{D})$ is the image by $\varphi$ of at most $p$ elements of $\mathbb{D}$.

Proof. To get the necessity, we could use Theorem 5.2 and the fact that $1-|z| \leq \log \frac{1}{|z|} \leq N_{\varphi}(\varphi(z))$; but we shall give a more elementary proof. Let $H M^{\Psi}$ be the closure of $H^{\infty}$ in $H^{\Psi}$. Since $C_{\varphi}\left(H^{\infty}\right) \subseteq H^{\infty}, C_{\varphi}$ maps $H M^{\Psi}$ into itself and $C_{\varphi}: H^{\Psi} \rightarrow H^{\Psi}$ being compact, its restriction $C_{\varphi}: H M^{\Psi} \rightarrow H M^{\Psi}$ is compact too. We know that the evaluation $\delta_{a}: f \in H M^{\Psi} \mapsto f(a) \in \mathbb{C}$ has norm $\approx \Psi^{-1}\left(\frac{1}{1-|a|}\right)\left([\mathbb{7}]\right.$, Lemma 3.11); hence $\delta_{a} /\left\|\delta_{a}\right\| \underset{|a| \rightarrow 1}{\longrightarrow} 0$ weak-star (because 
$\left|\delta_{a}(f)\right|=|f(a)| \leq\|f\|_{\infty}$ for $\left.f \in H^{\infty}\right)$. If $C_{\varphi}$ is compact, its adjoint $C_{\varphi}^{*}$ also; we get hence $\left\|C_{\varphi}^{*}\left(\delta_{a} /\left\|\delta_{a}\right\|\right)\right\| \underset{|a| \rightarrow 1}{\longrightarrow} 0$. But $C_{\varphi}^{*} \delta_{a}=\delta_{\varphi(a)}$. Therefore

$$
\frac{\Psi^{-1}\left(\frac{1}{1-|\varphi(a)|}\right)}{\Psi^{-1}\left(\frac{1}{1-|a|}\right)} \underset{|a| \rightarrow 1}{\longrightarrow} 0 .
$$

Conversely, assume that (5.6) holds. For every $A>0$, one has, for $|z|$ close enough to $1: \Psi^{-1}\left(\frac{1}{1-|z|}\right) \geq A \Psi^{-1}\left(\frac{1}{1-|\varphi(z)|}\right)$; in other words, one has: $1 / \Psi\left(A \Psi^{-1}(1 / 1-|\varphi(z)|)\right) \geq 1-|z|$. But, when $\varphi$ is $p$-valent, and if $w=\varphi(z)$ with $|z|>0$ minimal, one has $N_{\varphi}(w) \leq p \log \frac{1}{|z|} \approx 1-|z|$. Since $|z| \rightarrow 1$ when $|w|=|\varphi(z)| \rightarrow 1$ (otherwise, we should have a sequence $\left(z_{n}\right)$ converging to some $z_{0} \in \mathbb{D}$ and $\varphi\left(z_{n}\right)$ would converge to $\left.\varphi\left(z_{0}\right) \in \mathbb{D}\right)$, we get $\sup _{|w| \geq 1-h} N_{\varphi}(w) \lesssim$ $1 / \Psi\left(A \Psi^{-1}(1 / 1-|w|)\right) \leq 1 / \Psi\left(A \Psi^{-1}(1 / 1-h)\right)$, for $h$ small enough. By Theorem 5.2, with (5.4), that means that $C_{\varphi}$ is compact on $H^{\Psi}$.

Other consequences will be given in the subsequent paper [10].

Acknowledgement. This work has been initiated when the second-named author was invitated by the Departamento de Análisis Matemático of the Universidad de Sevilla, in April 2007; it is a pleasure to thanks all its members for their warm hospitality. Part of this work was also made during the fourth-named author visited the University of Lille 1 and the University of Artois (Lens) in June 2007. The fourth-named author is partially supported by a Spanish research project MTM2006-05622.

\section{References}

[1] L. Carleson, An interpolation problem for bounded analytic functions, Amer. J. Math. 80 (1958), 921-930.

[2] L. Carleson, Interpolations by bounded analytic functions and the corona problem, Annals of Math. (2) 76 (1962), 547-559.

[3] J. S. Choa and H.O. Kim, On function-theoretic conditions characterizing compact composition operators on $H^{2}$, Proc. Japan Acad. 75, Ser. A (1999), 109-112.

[4] B. R. Choe, The essential norms of composition operators, Glasg. Math. J. 34, No. 2 (1992), 143-155.

[5] M. Essén, D. F. Shea, and C. S. Stanton, A value-distribution criterion for the class $L \log L$ and some related questions, Ann. Inst. Fourier (Grenoble), 35 (1985), 125-150. 
[6] P. Lefèvre, D. Li, H. Queffélec, and L. Rodríguez-Piazza, Opérateurs de composition sur les espaces de Hardy-Orlicz, C. R. Math. Acad. Sci. Paris 344 (2007), no. 1, 5-10.

[7] P. Lefèvre, D. Li, H. Queffélec, and L. Rodríguez-Piazza, Composition operators on Hardy-Orlicz spaces, preprint, math.FA/0610905, to appear in Memoirs Amer. Math. Soc.

[8] P. Lefèvre, D. Li, H. Queffélec and L. Rodríguez-Piazza, Some examples of compact composition operators on $H^{2}$, Journal Funct. Anal. 255, No. 11 (2008), 3098-3124.

[9] P. Lefèvre, D. Li, H. Queffélec and L. Rodríguez-Piazza, Compact composition operators on $H^{2}$ and Hardy-Orlicz spaces, Journ. Math. Anal. Appl. 354 (2009), 360-371.

[10] P. Lefèvre, D. Li, H. Queffélec and L. Rodríguez-Piazza, Some revisited results about composition operators on Hardy spaces, preprint.

[11] J. E. Littlewood, On inequalities in the theory of functions, Proceedings London Math. Soc. (2) 23 (1925), 481-519.

[12] D. H. Luecking, Trace ideal criteria for Toeplitz operators, J. Funct. Anal. 73 (1987), 345-368.

[13] D. H. Luecking and K. Zhu, Composition operators belonging to the Schatten ideals, Amer. J. Math. 114 (1992), 878-906.

[14] B. McCluer, Compact composition operators on $H^{p}\left(B_{N}\right)$, Michigan Math. J. 32 (1985), 237-248.

[15] B. McCluer and J. Shapiro, Angular derivatives and compact composition operators on the Hardy and Bergman spaces, Canad. J. Math. 38, no. 4 (1986), 878-906.

[16] R. Nevanlinna, Le Théorème de Picard-Borel et la Théorie des Fonctions Méromorphes, second edition, Chelsea Publishing Company, New York (1974).

[17] S. C. Power, Vanishing Carleson measures, Bull. London Math. Soc. 12 (1980), no. 3, 207-210.

[18] L. A. Rubel, Entire and Meromorphic Functions, Universitext, Tracts in Mathematics, Springer-Verlag, New York (1996).

[19] J. H. Shapiro, Composition Operators and Classical Function Theory, Universitext, Tracts in Mathematics, Springer-Verlag, New York (1993).

[20] J. H. Shapiro, The essential norm of a composition operator, Annals of Math., 125 (1987), 375-404. 
[21] K. Zhu, Operator Theory in Function Spaces, Monographs and Textbooks in Pure and Applied Mathematics, 139, Marcel Dekker, Inc. (1990).

Pascal Lefèvre, Univ Lille Nord de France F-59 000 LILLE, U-Artois, Laboratoire de Mathématiques de Lens EA 2462, Fédération CNRS Nord-Pas-de-Calais FR 2956,

F-62 300 LENS, FRANCE

pascal.lefevre@euler.univ-artois.fr

Daniel Li, Univ Lille Nord de France F-59 000 LILLE,

U-Artois, Laboratoire de Mathématiques de Lens EA 2462,

Fédération CNRS Nord-Pas-de-Calais FR 2956,

F-62 300 LENS, Faculté des Sciences Jean Perrin,

Rue Jean Souvraz, S.P. 18, FRANCE

daniel.li@euler.univ-artois.fr

Hervé Queffélec, Univ Lille Nord de France F-59 000 LILLE,

USTL, Laboratoire Paul Painlevé U.M.R. CNRS 8524,

F-59 655 VILLENEUVE D'ASCQ Cedex, FRANCE

queff@math.univ-lille1.fr

Luis Rodríguez-Piazza, Universidad de Sevilla,

Facultad de Matemáticas, Departamento de Análisis Matemático,

Apartado de Correos 1160,

41080 SEVILLA, SPAIN

piazza@us.es 